\title{
10-羟基喜树碱氨基酸缀合物的合成及抗肿瘤活性研究
}

\author{
孟光荣 ${ }^{a}$ 李嘉俊 ${ }^{a}$ 王国林 ${ }^{b} \quad$ 董孟杰 ${ }^{b}$ 张 倩*,a \\ $\left({ }^{a}\right.$ 复旦大学药学院药物化学教研室 上海 201203) \\ ( ${ }^{b}$ 浙江大学附属一院 PET 中心 杭州 310003)
}

\begin{abstract}
摘要 以 10 -羊基喜树碱为原料, 通过两碳边链链接, 合成了一系列 10-羊基喜树碱氨基酸缀合物和 9-硝基-10-羊基喜 树碱氨基酸缀合物. 采用 CCK-8 法测试了合成化合物体外对人口腔鳞癌细胞 KB、人肝癌细胞 HepG2 和小鼠结肠癌细 胞 C26 三组细胞株的增殖抑制活性，结果表明部分目标化合物对所选肿瘤细胞株显示了潜在的抑制活性，其中 10-着基 喜树碱氨基酸缀合物的体外活性明显优于 9-硝基-10-羊基喜树碱氨基酸缀合物的体外活性.

关键词 喜树碱; 氨基酸缀合物; 抗肿瘤活性
\end{abstract}

\section{Synthesis and Cytotoxic Activities of the Amino Acid-Conjugates of 10-Hydroxycamptothecin}

\author{
Meng, Guangrong ${ }^{a} \quad$ Li, Jiajun ${ }^{a} \quad$ Wang, Guolin $^{b} \quad$ Dong, Mengjie ${ }^{b} \quad$ Zhang, Qian*,a \\ ( ${ }^{a}$ Department of Medicinal Chemistry, School of Pharmacy, Fudan University, Shanghai 201203) \\ ( ${ }^{b}$ Positron Emission Tomography Center, First Affiliated Hospital, Zhejiang University, Hangzhou 310003)
}

\begin{abstract}
A series of amino acid-conjugates of 10-hydroxycamptothecin and 9-nitro-10-hydroxycamptothecin were synthesized by means of a two-carbon side chain from 10-hydroxycamptothecin. The cytotoxic activities of all the synthetic compounds on KB, HepG2 and C26 in vitro were evaluated by CCK-8 assay. The results revealed that part of the conjugates exhibit potent anti-proliferative activities against selected tumor cell lines. Among them, the amino acid-conjugates of 10-hydroxycamptothecin show more potent activities than those of 9-nitro-10-hydroxycamptothecin in vitro as antitumor agents.
\end{abstract}

Keywords camptothecin; amino acid-conjugates; cytotoxic activities

喜树碱(Camptothecin, CPT)是 1966 年由 Wall 和 Wani 等 ${ }^{[1]}$ 首次从中国特有珙桐科植物喜树 (Camptotheca acuminate) 中提取得到的一种具有拼合五环结构的生物 碱. 该类化合物主要通过与 DNA及拓扑异构酶 I 作用形 成三元复合物, 阻滞 DNA 复制, 导致细胞死亡, 从而显 示出显著的抗肿瘤活性 ${ }^{[2 \sim 6]}$. 其中, 10-羟基喜树碱 (10-Hydoxycamptothecin, HCPT, 1) 是天然的喜树碱衍生 物之一, 20 世纪 60 年代末我国即开展对 10 -喜树碱类化 合物的研究工作, 70 年代 10-羊基喜树碱的临床试验在 国内开始, 1988 年获准上市 ${ }^{[7,8]}$, 主要用于胃癌、肝癌、 头颈部癌及白血病治疗. 目前已被列入国家基本药物及 医保用药目录，是我国特有的自行研制开发的天然抗肿 瘤药物.
但是，10-羊基喜树碱溶解性较差和毒副作用较大 等缺点严重影响了其临床应用 ${ }^{[0 ~ 12]}$. 为克服这些缺点, 我们利用前药原理 ${ }^{[13,14]}$, 通过两碳边链连接, 引入不同 的氨基酸片段，设计了一系列 10-羊基喜树碱氨基酸缀 合物(图 1). 期望氨基酸片段的引入, 不仅可以改善喜树 碱类化合物的水溶性，同时也能够提高化合物对肿瘤细 胞的靶向性.

喜树碱 $\mathrm{E}$ 环开环是引起该类化合物毒副作用的主 要原因, 有文献报道 ${ }^{[15]}, 9$ 位基团的引入有益于 $\mathrm{E}$ 环的 稳定性，因此，我们也尝试合成了一系列 9-硝基-10-羊杢 基喜树碱的氨基酸缀合物.

\footnotetext{
*E-mail: zhangqian511@shmu.edu.cn

Received August 4, 2013; revised August 30, 2013; published online September 17, 2013.

Project supported by the National Natural Science Foundation of China (No. 30870730).

国家自然科学基金(No. 30870730)资助项目.
} 


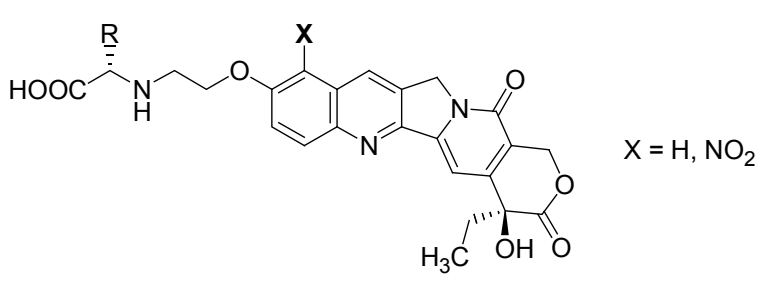

图 1 10-羊基喜树碱氨基酸缀合物的设计

Figure 1 Design of amino acid-conjugates of camptothecin

\section{1 结果与讨论}

微波催化条件下，10-差基喜树碱与 1,2-二溴乙烷反 应生成 10-(2-溴乙氧基)喜树碱(2), 化合物 2 分别与脯氨 酸、酪氨酸甲酯、苯丙氨酸、甲硫氨酸、缅氨酸、苯丙 氨酸甲酯以及甘氨酸乙酯的氨基发生亲核取代反应，相 应地生成 $4 \mathrm{a} \sim 4 \mathrm{~g}$ 等 7 个 10 -羟基喜树碱的氨基酸缀合物; 10-(2-溴乙氧基)喜树碱(2)在混酸作用下, 引入 9-硝基, 9-硝基-10-(2-溴乙氧基)喜树碱(3)分别与脯氨酸和酪氨 酸甲酯缩合，生成 $5 \mathbf{a}$ 和 $\mathbf{5 b}$ (Scheme 1).

在分离和纯化化合物 $4 \mathrm{a}, 4 \mathrm{c} \sim 4 \mathrm{e}$ 的过程中, 同时分 离得到了这类化合物的水解产物 10-(2-羟乙氧基)喜树 碱(6). 我们推测其原因, 认为与氨基酸片段以内盐形式 存在相关, 氮正离子的形成增加了碳氮键断裂的可能
性，从而促进了水解反应发生，导致这类化合物的收率 普遍较低

而对于氨基酸酯缀合物 $4 \mathrm{~b}, 4 \mathrm{f}, 4 \mathrm{~g}$ 以及 $5 \mathrm{~b}$ ，反应过 程中并未获得水解产物，反应收率也相对较高(19\% 40\%), 从而进一步验证了氨基酸片段内盐对化合物稳 定性影响的推理.

我们利用 Molinspiration 软件(网络版) $)^{[16,17]}$ 对所得 目标化合物的脂水分配系数 $(\log P)$ 进行了预测，计算值 见表 1. 计算结果显示, (1)目标分子中, 羧基游离的氨基 酸缀合物的 $\log P$ 均小于 10 -差基喜树碱的 $\log P$ 值, 说 明氨基酸的引入可以明显增强该类化合物的水溶性，这 与我们在实验操作过程中对化合物的认识是一致的. (2) 羧基末端成酯的氨基酸缀合物的 $\log P$ 明显大于羧基游 离的氨基酸缀合物，同时也大于 10 -差基喜树碱，预测 羧基末端成酯后可能会降低化合物的水溶性. (3)通过 5a 与 $4 \mathrm{a}, 5 \mathrm{~b}$ 与 $4 \mathrm{~b}$ 的预测值对比可以发现, 9 位硝基的引入, 可能对化合物的溶解性影响不大.

以 10-羊基喜树碱为阳性对照, 分别测试了 9 个目 标分子、 2 个中间体以及化合物 6 的体外抗肿瘤细胞增 殖活性(见表 2). $\mathrm{IC}_{50}$ 数据显示, (1) 10-(2-溴乙氧基)喜树 碱(2)和 10-(2-羊至乙氧基)喜树碱(6)体外活性与 10-羊基喜

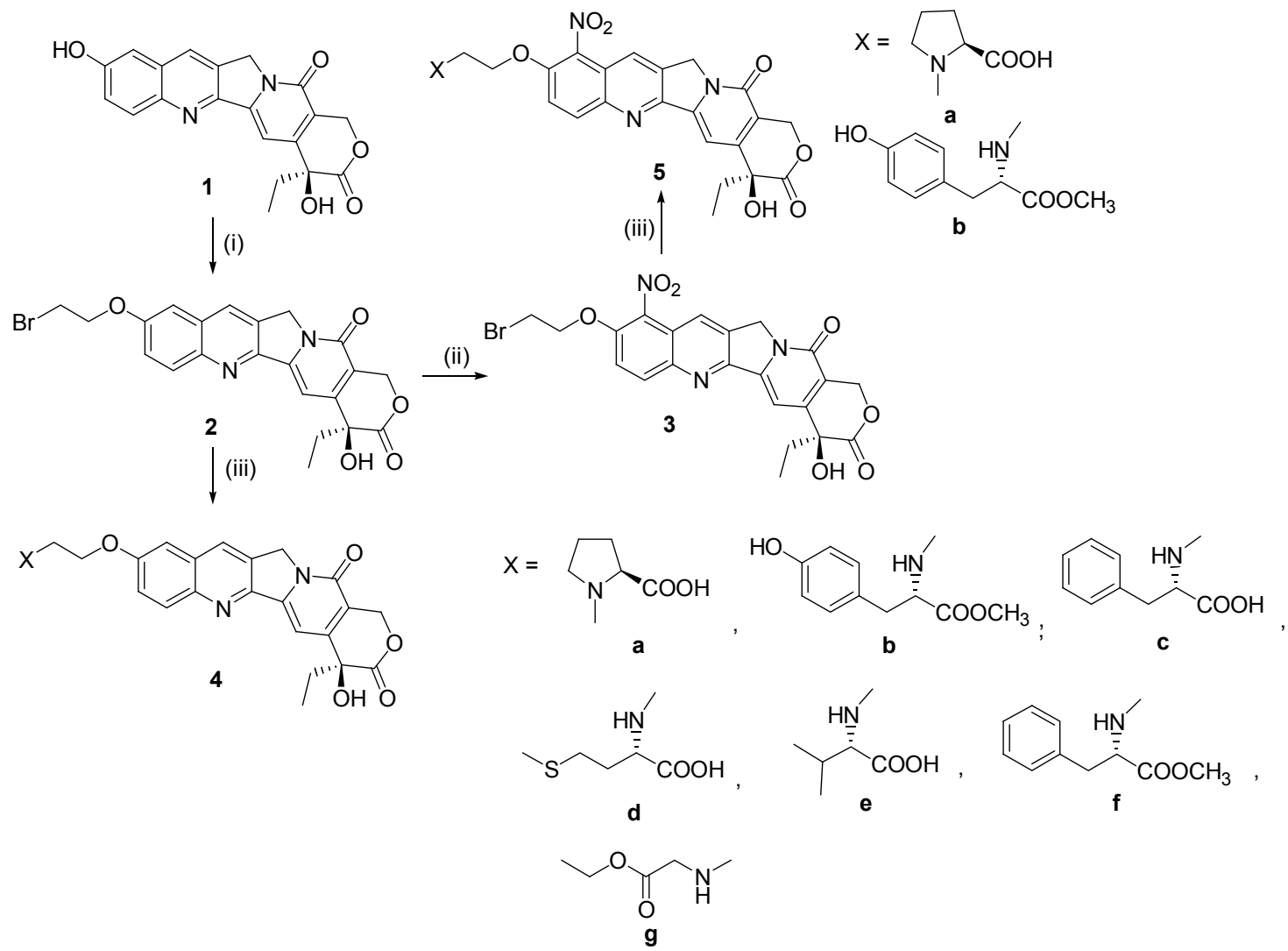

Reagents and conditions: (i) $\mathrm{BrCH}_{2} \mathrm{CH}_{2} \mathrm{Br}, \mathrm{K}_{2} \mathrm{CO}_{3}$, DMSO, MW, $60{ }^{\circ} \mathrm{C}, 52.8 \%$; (ii) $\mathrm{H}_{2} \mathrm{SO}_{4}(98 \%), \mathrm{HNO}_{3}(65 \%), 60.9 \%$; (iii) Amino acid, DMSO, $80{ }^{\circ} \mathrm{C}, 6.5 \% \sim 40.2 \%$.

\section{Scheme 1}




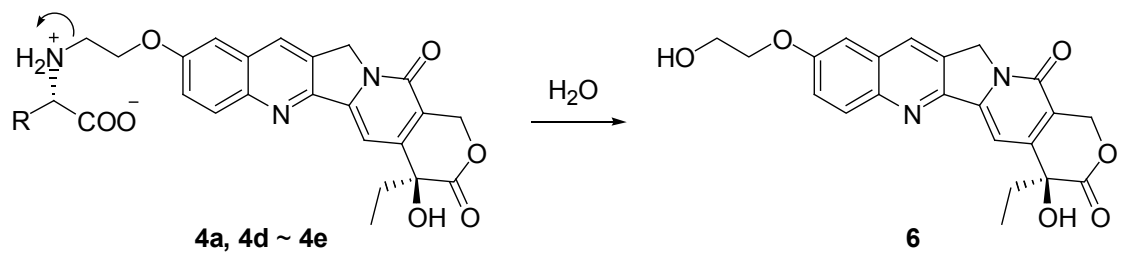

图 2 10-羊基喜树碱氨基酸缀合物的水解

Figure 2 Hydrolysis of amino acid-conjugates of 10-hydroxycampothecin

表 1 10-羊基喜树碱-氨基酸缀合物 $\log P$ 计算值

Table 1 Calculated $\log P$ for amino acid-conjugates of 10-hydroxycamptothecin

\begin{tabular}{cccccccccccccc}
\hline Compd. & HCPT & $\mathbf{2}$ & $\mathbf{4 a}$ & $\mathbf{4 b}$ & $\mathbf{4 c}$ & $\mathbf{4 d}$ & $\mathbf{4 e}$ & $\mathbf{4 f}$ & $\mathbf{4 g}$ & $\mathbf{3}$ & $\mathbf{5 a}$ & $\mathbf{5 b}$ & $\mathbf{6}$ \\
\hline $\log P$ & 1.53 & 2.80 & 0.27 & 3.02 & 1.43 & 0.42 & 0.74 & 3.50 & 1.73 & 2.99 & 0.46 & 3.02 & 1.43 \\
\hline
\end{tabular}

树碱相当; (2) 10-羊基喜树碱的氨基酸缀合物 $\mathbf{4 a} \sim \mathbf{4 g}$ 均 保持了喜树碱类化合物显著的抗肿瘤活性, 其中羧基游 离的氨基酸缀合物的活性略高于羧基成酯的氨基酸酯 缀合物的活性, 尤其是缴氨酸缀合物 $4 \mathrm{e}$ 活性水平与 10 羟基喜树碱相近; (3) 9-位引入硝基的化合物活性均有丧 失, 对 C26 细胞株的抑制活性, 3 比 2 降低了近 8 倍, 化 合物 5a 和 $\mathbf{5 b}$ 与 10-羊基喜树碱比较, 活性降低了近 100 倍, 从而说明在该类结构中，9 位硝基对目标化合物的 抑制肿瘤细胞增殖活性不利.

表 2 10-羊基喜树碱氨基酸缀合物的体外细胞毒活性

Table 2 Cytotoxic activities of the amino acid-conjugates of 10-hydroxycamptothecin in vitro

\begin{tabular}{cccc}
\hline \multirow{2}{*}{ Compd. } & \multicolumn{3}{c}{$\mathrm{IC}_{50} /\left(\mu \mathrm{mol} \cdot \mathrm{L}^{-1}\right)$} \\
\cline { 2 - 4 } & $\mathrm{C} 26$ & $\mathrm{HepG} 2$ & $\mathrm{~KB}$ \\
\hline $\mathrm{HCPT}$ & 0.042 & 0.553 & 1.475 \\
$\mathbf{2}$ & 0.079 & 0.544 & 6.656 \\
$\mathbf{4 a}$ & 0.101 & 0.883 & 3.670 \\
$\mathbf{4 b}$ & 1.636 & 4.514 & 2.416 \\
$\mathbf{4} \mathbf{c}$ & 0.138 & 0.813 & 4.069 \\
$\mathbf{4 d}$ & 0.155 & 1.109 & 1.947 \\
$\mathbf{4 e}$ & 0.076 & 0.580 & 1.388 \\
$\mathbf{4 f}$ & 0.950 & 3.918 & 5.556 \\
$\mathbf{4 g}$ & 0.438 & 2.694 & 4.760 \\
$\mathbf{3}$ & 0.664 & 2.499 & 10.659 \\
$\mathbf{5 a}$ & 6.065 & 85.90 & 71.02 \\
$\mathbf{5 b}$ & 6.719 & 60.45 & 29.62 \\
$\mathbf{6}$ & 0.095 & 0.395 & 1.506 \\
\hline
\end{tabular}

\section{2 结论}

通过 10-羊基醚化形成的两碳边链, 将 10-羊基喜树 碱和不同氨基酸链接起来, 合成了一系列 10-羊基喜树 碱氨基酸缀合物和 9-硝基-10-羊基喜树碱氨基酸缀合 物. 部分缀合物显示了体外对人口腔鳞癌细胞 $\mathrm{KB}$ 、人 肝癌细胞 HepG2 和小鼠结肠癌细胞 C26 显著的抑制活 性, 其中缬氨酸与 10-羊基喜树碱的缀合物 $\mathbf{4 e}$ 不仅保持
了与 10-差基喜树碱相当的抑制肿瘤增殖活性，同时其 水溶性也较 10-差基喜树碱有了明显的提高，值得进一 步深入研究.

\section{3 实验部分}

\section{1 仪器与试剂}

10-羟基喜树碱购自成都天源天然产物有限公司, 其他所有实验用试剂均为市售国产分析纯; ${ }^{1} \mathrm{H}$ NMR 谱 用 Bruker-DPX $400 \mathrm{MHz}$ 核磁共振仪测定(以 DMSO- $d_{6}$ 为溶剂, 以 $\mathrm{TMS}$ 为内标); 质谱用 Agilent G1946D 质谱 仪测定; 高分辨质谱用 Bruker APEXIII 7.0 TESLA FTMS 测定; 熔点用 SGW X-4 显微熔点仪测定; TLC 用 板为青岛海洋 $0.2 \mathrm{~mm} \mathrm{GF} 254$ 高效薄层层析硅胶板; 微 波反应仪为祥鹄 XH-100B 型微波催化合成仪.

\section{2 化合物合成}

\subsubsection{0-(2-溴乙氧基)喜树碱(2)的合成}

10-羊基喜树碱 $50 \mathrm{mg}$ 于 $10 \mathrm{~mL}$ 微波反应瓶中, 加入 $2 \mathrm{~mL}$ DMSO 微加热溶清, 依次加入 3 倍量无水碳酸钾 和 7 倍量 1,2-二溴乙烷, 置于微波反应仪中. 磁力搅拌 下于 $600 \mathrm{~W}$ 和 $60{ }^{\circ} \mathrm{C}$ 下反应 $5 \mathrm{~min}$, 自然冷却至室温, 将 反应液慢慢滴加到 $40 \mathrm{~mL}$ 冰水中, 过滤得到淡黄色固体 粗品. 粗品用甲醇/氯仿混合溶剂溶清, 柱层析 [Silica Gel $\mathrm{H}$, 洗脱剂: $V$ (二氯甲烷) $: V$ (甲醇 $)=35: 1]$, 分得 淡黄色粉末状固体 $234.1 \mathrm{mg}$, m.p. 262 264 ${ }^{\circ} \mathrm{C}$, 收率 $52.8 \%{ }^{[18]}$. ${ }^{1} \mathrm{H}$ NMR (DMSO- $\left.d_{6}, 400 \mathrm{MHz}\right) \delta: 0.86(\mathrm{t}, J=$ $\left.7.24 \mathrm{~Hz}, 3 \mathrm{H}, 18-\mathrm{CH}_{3}\right), 1.84 \sim 1.86\left(\mathrm{~m}, 2 \mathrm{H}, 19-\mathrm{CH}_{2}\right), 3.90$ (t, $\left.J=5.28 \mathrm{~Hz}, 2 \mathrm{H}, \mathrm{Br}-\mathrm{CH}_{2}\right), 4.49$ (t, $J=5.28 \mathrm{~Hz}, 2 \mathrm{H}$, $\left.10-\mathrm{O}-\mathrm{CH}_{2}\right), 5.23\left(\mathrm{~s}, 2 \mathrm{H}, 5-\mathrm{CH}_{2}\right), 5.40\left(\mathrm{~s}, 2 \mathrm{H}, 17-\mathrm{CH}_{2}\right)$, $6.50(\mathrm{~s}, 1 \mathrm{H}, 20-\mathrm{OH}), 7.26(\mathrm{~s}, 1 \mathrm{H}, 14-\mathrm{CH}), 7.51$ (dd, $J=$ 9.39, $2.74 \mathrm{~Hz}, 1 \mathrm{H}, 11-\mathrm{CH}), 7.54$ (d, $J=2.74 \mathrm{~Hz}, 1 \mathrm{H}$, 9-CH), 8.12 (d, J=9.39 Hz, 1H, 12-CH), 8.50 (s, 1H, 7-CH); ESI-MS m/z: $471.0\left(\mathrm{M}+\mathrm{H}^{+}\right), 473.0\left(\mathrm{M}+2+\mathrm{H}^{+}\right)$. 


\subsubsection{9-硝基-10-(2-溴乙氧基)喜树碱(3)的合成}

冰水浴条件下, $100 \mathrm{mg}$ (0.21 mmol) 2 缓慢加至 4 $\mathrm{mL} 98 \%$ 硫酸中, 搅拌 $15 \mathrm{~min}$ 后底物溶清, 缓慢滴加冷 却的混酸 $0.8 \mathrm{~mL}[V(98 \% \text { 硫酸 }): V(65 \% \text { 硝酸 })=1: 1]^{[19]}$. $25 \mathrm{~min}$ 后将反应液滴加到 $40 \mathrm{~mL}$ 冰水中并剧烈搅拌. 氯 仿/甲醇萃取, 有机相水洗至中性、饱和食盐水洗涤、无 水硫酸钠干燥, 过滤, 滤液蒸除溶剂后, 得到 $98.3 \mathrm{mg}$ 粗 品. 粗品用甲醇/氯仿混合溶剂溶清, 柱层析 [Silica Gel $\mathrm{H}$, 洗脱剂: $V$ (二氯甲烷 $): V($ 甲醇 $)=40: 1]$, 分得化合 物 3, 黄色固体, m.p. $270 \sim 273{ }^{\circ} \mathrm{C}$, 收率 $60.9 \%$. ${ }^{1} \mathrm{H}$ NMR (DMSO- $\left.d_{6}, 400 \mathrm{MHz}\right) \delta: 0.86$ (t, $J=7.14 \mathrm{~Hz}, 3 \mathrm{H}$, $\left.18-\mathrm{CH}_{3}\right), 1.82 \sim 1.89\left(\mathrm{~m}, 2 \mathrm{H}, 19-\mathrm{CH}_{2}\right), 3.84(\mathrm{t}, J=5.16$ $\left.\mathrm{Hz}, 2 \mathrm{H}, \mathrm{Br}-\mathrm{CH}_{2}\right), 4.74$ (t, J=4.96 Hz, 2H, 10-OCH $), 5.26$ (s, 2H, 5- $\left.\mathrm{CH}_{2}\right), 5.42\left(\mathrm{~s}, 2 \mathrm{H}, 17-\mathrm{CH}_{2}\right), 6.54(\mathrm{~s}, 1 \mathrm{H}, 20-\mathrm{OH})$, 7.32 (s, 1H, 14-CH), 8.03 (d, J=9.51 Hz, 1H, 11-CH), $8.42(\mathrm{~d}, J=9.52 \mathrm{~Hz}, 1 \mathrm{H}, 12-\mathrm{CH}), 8.46$ (s, 1H, 7-CH); ESI-MS $m / z$ : $514.1\left(\mathrm{M}-\mathrm{H}^{+}\right), 516.1\left(\mathrm{M}+2-\mathrm{H}^{+}\right)$

\subsubsection{0-喜树碱氨基酸缀合物 $\mathbf{4 a} \sim \mathbf{4 g}$ 的合成}

$100 \mathrm{mg}$ (0.21 mmol)化合物 2 于圆底烧瓶中, 溶于 2 $\mathrm{mL}$ DMSO, 加入 1.5 倍量 $L$-氨基酸类试剂, 外浴 $80{ }^{\circ} \mathrm{C}$ 下反应, 反应时间 $3 \sim 8 \mathrm{~h}$ 不等. 反应完全后, 自然冷却 至室温, 将反应液慢慢滴加到 $40 \mathrm{~mL}$ 冰水中, 过滤得到 黄色固体粗品. 粗品用甲醇/氯仿混合溶剂溶清, 柱层析 [Silica Gel H, 洗脱剂: $V$ (二氯甲烷 $): V$ 甲醇 $)=30 ： 1$ ], 分得目标化合物 $\mathbf{4 a} \sim \mathbf{4 g}$.

10-差基喜树碱脯氨酸缀合物(4a): 黄色固体, m.p. $227 \sim 230{ }^{\circ} \mathrm{C}$; 收率 22.20\%. ${ }^{1} \mathrm{H}$ NMR (DMSO- $d_{6}, 400$ $\mathrm{MHz}) \delta: 0.87\left(\mathrm{t}, J=7.24 \mathrm{~Hz}, 3 \mathrm{H}, 18-\mathrm{CH}_{3}\right), 1.80 \sim 1.92(\mathrm{~m}$, $4 \mathrm{H}, 19-\mathrm{CH}_{2}$ and $\left.4^{\prime}-\mathrm{CH}_{2}\right), 1.94 \sim 1.99\left(\mathrm{~m}, 1 \mathrm{H}, 3^{\prime}-\mathrm{CH}_{2}{ }^{\prime}\right)$, $2.17 \sim 2.25\left(\mathrm{~m}, 1 \mathrm{H}, 3^{\prime}-\mathrm{CH}_{2}{ }^{\prime}\right), 3.06 \sim 3.34\left(\mathrm{~m}, 2 \mathrm{H}, 5^{\prime}-\mathrm{CH}_{2}\right.$ and $\mathrm{OCH}_{2} \mathrm{CH}_{2}$ ), 4.30 (t, $J=7.82 \mathrm{~Hz}, 1 \mathrm{H}, 2^{\prime}-\mathrm{CH}$ ), 4.43 (brs, $2 \mathrm{H}, \quad 5^{\prime}-\mathrm{CH}_{2}{ }^{\prime}$ and $\left.\mathrm{O}-\mathrm{CH}_{2} \mathrm{CH}_{2}{ }^{\prime}\right), 4.55 \sim 4.63(\mathrm{~m}, 2 \mathrm{H}$, $\left.\mathrm{OCH}_{2} \mathrm{CH}_{2}\right), 5.26\left(\mathrm{~s}, 2 \mathrm{H}, 5-\mathrm{CH}_{2}\right), 5.41\left(\mathrm{~s}, 2 \mathrm{H}, 17-\mathrm{CH}_{2}\right)$, 6.52 (s, 1H, 20-OH), 7.27 (s, 1H, 14-CH), 7.50 (d, $J=9.39$ $\mathrm{Hz}, 1 \mathrm{H}, 11-\mathrm{CH}), 7.58$ (s, 1H, 9-CH), 8.08 (d, $J=9.39 \mathrm{~Hz}$, 1H, 12-CH), 8.53 (s, 1H, 7-CH); ESI-MS m/z: $506.0(\mathrm{M}+$ $\mathrm{H}^{+}$); HRMS calcd for $\mathrm{C}_{27} \mathrm{H}_{28} \mathrm{~N}_{3} \mathrm{O}_{7}[\mathrm{M}+\mathrm{H}]^{+}$506.1922, found 506.1940.

10-羊基喜树碱酪氨酸甲酯缀合物(4b): 黄色固体, m.p. $128 \sim 132{ }^{\circ} \mathrm{C}$; 收率 $30.40 \%$. ${ }^{1} \mathrm{H}$ NMR (DMSO- $d_{6}$, $400 \mathrm{MHz}) \delta: 0.86\left(\mathrm{t}, J=7.24 \mathrm{~Hz}, 3 \mathrm{H}, 18-\mathrm{CH}_{3}\right), 1.81 \sim 1.88$ (m, 2H, 19- $\mathrm{CH}_{2}$ ), 2.21 (brs, 1H, NH), 2.76 (brs, 2H, $\left.\mathrm{NHCH}_{2}\right), 2.87 \sim 2.95\left(\mathrm{~m}, 2 \mathrm{H}, \mathrm{PhCH}_{2}\right), 3.52(\mathrm{~s}, 4 \mathrm{H}$, $\mathrm{NHCH}-\mathrm{COOCH}_{3}$ ), 4.13 (brs, 2H, 10-OCH $), 5.24$ (s, 2H, $\left.5-\mathrm{CH}_{2}\right), 5.40\left(\mathrm{~s}, 2 \mathrm{H}, 17-\mathrm{CH}_{2}\right), 6.49(\mathrm{~s}, 1 \mathrm{H}, 20-\mathrm{OH}), 6.62$ (d, $\left.J=8.22 \mathrm{~Hz}, \mathrm{Ph}-3{ }^{\prime}, 5^{\prime}-\mathrm{CH}\right), 6.94$ (d, $J=8.22 \mathrm{~Hz}$, Ph-2',6'-CH), 7.26 (s, 1H, 14-CH), 7.43-7.47 (m, 2H, $11-\mathrm{CH}$ and 9-CH ), $8.04(\mathrm{~d}, J=9.39 \mathrm{~Hz}, 1 \mathrm{H}, 12-\mathrm{CH}), 8.50$ (s, 1H, 7-CH), 9.18 (s, 1H, Ph-OH); ESI-MS m/z: 586.2 $\left(\mathrm{M}+\mathrm{H}^{+}\right)$; HRMS calcd for $\mathrm{C}_{32} \mathrm{H}_{32} \mathrm{~N}_{3} \mathrm{O}_{8}[\mathrm{M}+\mathrm{H}]^{+}$ 586.2184, found 586.2175.

10-羊基喜树碱苯丙氨酸缀合物(4c): 淡黄色固体, m.p. $178 \sim 181{ }^{\circ} \mathrm{C}$; 收率: $27.15 \% .{ }^{1} \mathrm{H}$ NMR (DMSO- $d_{6}$, $400 \mathrm{MHz}) \delta: 0.83\left(\mathrm{t}, J=7.05 \mathrm{~Hz}, 3 \mathrm{H}, 18-\mathrm{CH}_{3}\right), 1.81 \sim 1.90$ (m, 2H, 19- $\mathrm{CH}_{2}$ ), 2.31 (brs, $\left.1 \mathrm{H}, \mathrm{NH}\right), 2.81 \sim 2.84(\mathrm{~m}, 2 \mathrm{H}$, $\left.\mathrm{NHCH}_{2}\right), 4.16(\mathrm{t}, J=5.08 \mathrm{~Hz}, 1 \mathrm{H}, \mathrm{NHCH}), 4.30 \sim 4.32$ $\left(\mathrm{m}, 2 \mathrm{H}, \mathrm{PhCH}_{2}\right), 4.40 \sim 4.42\left(\mathrm{~m}, 2 \mathrm{H}, 10-\mathrm{OCH}_{2}\right), 5.25(\mathrm{~s}$, $\left.2 \mathrm{H}, 5-\mathrm{CH}_{2}\right), 5.40\left(\mathrm{~s}, 2 \mathrm{H}, 17-\mathrm{CH}_{2}\right), 6.50(\mathrm{~s}, 1 \mathrm{H}, 20-\mathrm{OH})$, $7.10 \sim 7.13\left(\mathrm{~m}, 5 \mathrm{H}, \mathrm{CH}_{2} \mathbf{P h}\right), 7.27(\mathrm{~s}, 1 \mathrm{H}, 14-\mathrm{CH}), 7.48 \sim$ $7.52(\mathrm{~m}, 2 \mathrm{H}, 9-\mathrm{CH}, 11-\mathrm{CH}), 8.06$ (d, $J=9.00 \mathrm{~Hz}, 1 \mathrm{H}$, 12-CH), 8.52 (s, 1H, 7-CH); ESI-MS m/z: $556.2\left(\mathrm{M}+\mathrm{H}^{+}\right)$; HRMS calcd for $\mathrm{C}_{31} \mathrm{H}_{30} \mathrm{~N}_{3} \mathrm{O}_{7}[\mathrm{M}+\mathrm{H}]^{+}$556.2078, found 556.2076 .

10-羊基喜树碱甲硫氨酸缀合物 (4d): 黄色固体, m.p. $175 \sim 178{ }^{\circ} \mathrm{C}$, 收率 $18.80 \%$. ${ }^{1} \mathrm{H}$ NMR (DMSO- $d_{6}$, $400 \mathrm{MHz}) \delta: 0.86\left(\mathrm{t}, J=7.04 \mathrm{~Hz}, 3 \mathrm{H}, 18-\mathrm{CH}_{3}\right), 1.81 \sim 1.88$ (m, $J=7.05 \mathrm{~Hz}, 2 \mathrm{H}, 19-\mathrm{CH}_{2}$ ), 1.96 (brs, 2H, S-CH $\mathrm{CH}_{2}$ ), 2.02 (s, 3H, $\mathrm{SCH}_{3}$ ), 2.48 (brs, 4H, S-CH, $\mathrm{NHCH}_{2}$ ), 4.14 $(\mathrm{t}, J=6.26 \mathrm{~Hz}, 1 \mathrm{H}, \mathrm{NHCH}), 4.58 \sim 4.64(\mathrm{~m}, 2 \mathrm{H}, 10-$ $\left.\mathrm{OCH}_{2}\right), 5.27\left(\mathrm{~s}, 2 \mathrm{H}, 5-\mathrm{CH}_{2}\right), 5.41\left(\mathrm{~s}, 2 \mathrm{H}, 17-\mathrm{CH}_{2}\right), 6.52(\mathrm{~s}$, $1 \mathrm{H}, 20-\mathrm{OH}), 7.27(\mathrm{~s}, 1 \mathrm{H}, 14-\mathrm{CH}), 7.46 \sim 7.50(\mathrm{~m}, 2 \mathrm{H}$, 9-CH, 11-CH), 8.09 (d, $J=9.39 \mathrm{~Hz}, 1 \mathrm{H}, 12-\mathrm{CH}), 8.54$ (s, 1H, 7-CH); ESI-MS m/z: $540.2\left(\mathrm{M}+\mathrm{H}^{+}\right)$; HRMS calcd for $\mathrm{C}_{27} \mathrm{H}_{30} \mathrm{~N}_{3} \mathrm{O}_{7} \mathrm{~S}[\mathrm{M}+\mathrm{H}]^{+}$540.1799, found 540.1802.

10-差基喜树碱缬氨酸缀合物(4e): 黄色固体, m.p. 209 211 ${ }^{\circ} \mathrm{C}$, 收率 11.12\%. ${ }^{1} \mathrm{H}$ NMR (DMSO- $d_{6}, 400$ $\mathrm{MHz}) \delta: 0.84 \sim 0.88\left(\mathrm{~m}, 9 \mathrm{H}, 18-\mathrm{CH}_{3}, \mathrm{CH}\left(\mathrm{CH}_{3}\right)_{2}\right), 1.81 \sim$ $1.88\left(\mathrm{~m}, 2 \mathrm{H}, 19-\mathrm{CH}_{2}\right), 2.04 \sim 2.07\left(\mathrm{~m}, 1 \mathrm{H},\left(\mathrm{CH}_{3}\right)_{2} \mathrm{CH}\right)$, $3.80\left(\mathrm{t}, J=4.70 \mathrm{~Hz}, 2 \mathrm{H}, \mathrm{NCH}_{2}\right), 4.15(\mathrm{t}, J=4.69 \mathrm{~Hz}, 2 \mathrm{H}$, $\left.\mathrm{OCH}_{2}\right), 4.97(\mathrm{t}, J=5.09 \mathrm{~Hz}, 1 \mathrm{H}, \mathrm{NHCH}), 5.26(\mathrm{~s}, 2 \mathrm{H}$, 5- $\left.\mathrm{CH}_{2}\right), 5.40$ (s, 2H, 17- $\left.\mathrm{CH}_{2}\right), 6.51(\mathrm{~s}, 1 \mathrm{H}, 20-\mathrm{OH}), 7.27$ (s, $1 \mathrm{H}, 14-\mathrm{CH}$ ), $7.51 \sim 7.54$ (m, 2H, 9-CH, 11-CH), 8.07 (d, $J=9.00 \mathrm{~Hz}, 1 \mathrm{H}, 12-\mathrm{CH}), 8.52$ (s, $1 \mathrm{H}, 7-\mathrm{CH})$; ESI-MS $m / z$ : $508.2\left(\mathrm{M}+\mathrm{H}^{+}\right)$; HRMS calcd for $\mathrm{C}_{27} \mathrm{H}_{29} \mathrm{~N}_{3} \mathrm{O}_{7}[\mathrm{M}+\mathrm{H}]^{+}$ 508.2078, found 508.2079.

10-羊基喜树碱苯丙氨酸甲酯缀合物(4f)：淡黄色固 体, m.p. 186 $189{ }^{\circ} \mathrm{C}$, 收率 40.17\%. ${ }^{1} \mathrm{H}$ NMR (DMSO$\left.d_{6}, 400 \mathrm{MHz}\right) \delta: 0.86\left(\mathrm{t}, J=7.24 \mathrm{~Hz}, 3 \mathrm{H}, 18-\mathrm{CH}_{3}\right), 1.81 \sim$ $1.88\left(\mathrm{~m}, 2 \mathrm{H}, 19-\mathrm{CH}_{2}\right), 2.31$ (brs, $\left.1 \mathrm{H}, \mathrm{NH}\right), 2.84 \sim 2.88(\mathrm{~m}$, $\left.4 \mathrm{H}, \mathrm{NHCH}_{2}, \mathrm{PhCH}_{2}\right), 3.53$ (s, 3H, $\left.\mathrm{COOCH}_{3}\right), 3.61$ (brs, 
1H, NHCH), 4.14 (brs, 2H, 10- $\left.-\mathrm{OCH}_{2}\right), 5.25$ (s, 2H, 5- $\left.\mathrm{CH}_{2}\right), 5.40\left(\mathrm{~s}, 2 \mathrm{H}, 17-\mathrm{CH}_{2}\right), 6.53(\mathrm{~s}, 1 \mathrm{H}, 20-\mathrm{OH}), 7.17 \sim$ $7.25\left(\mathrm{~m}, 5 \mathrm{H}, \mathrm{CH}_{2} \mathrm{Ph}\right), 7.26(\mathrm{~s}, 1 \mathrm{H}, 14-\mathrm{CH}), 7.44 \sim 7.48(\mathrm{~m}$, 2H, 9-CH, 11-CH), 8.05 (d, $J=9.00 \mathrm{~Hz}, 1 \mathrm{H}, 12-\mathrm{CH}), 8.51$ (s, 1H, 7-CH). ESI-MS m/z: $570.2\left(\mathrm{M}+\mathrm{H}^{+}\right)$; HRMS calcd for $\mathrm{C}_{32} \mathrm{H}_{32} \mathrm{~N}_{3} \mathrm{O}_{7}[\mathrm{M}+\mathrm{H}]^{+}$570.2235, found 570.2230.

10-羊基喜树碱甘氨酸乙酯缀合物 (4g): 淡黄色固 体, m.p. 168 $170{ }^{\circ} \mathrm{C}$, 收率 19.40\%. ${ }^{1} \mathrm{H}$ NMR (DMSO$\left.d_{6}, 400 \mathrm{MHz}\right) \delta: 0.86\left(\mathrm{t}, J=7.24 \mathrm{~Hz}, 3 \mathrm{H}, 18-\mathrm{CH}_{3}\right), 1.82 \sim$ $1.86\left(\mathrm{~m}, 2 \mathrm{H}, 19-\mathrm{CH}_{2}\right), 2.99$ (t, $J=5.28 \mathrm{~Hz}, 2 \mathrm{H}, \mathrm{NHCH}_{2}$ ), $3.43\left(\mathrm{~s}, 2 \mathrm{H}, \mathrm{NHCH}_{2} \mathrm{CO}\right), 3.53 \sim 3.57(\mathrm{~m}, 3 \mathrm{H}, \mathrm{COO}-$ $\left.\mathrm{CH}_{2} \mathrm{CH}_{3}\right), 4.04 \sim 4.10\left(\mathrm{~m}, 2 \mathrm{H}, \mathrm{COOCH} \mathbf{H}_{2}\right), 4.18(\mathrm{t}, J=5.29$ $\left.\mathrm{Hz}, 2 \mathrm{H}, 10-\mathrm{OCH}_{2}\right), 5.25\left(\mathrm{~s}, 2 \mathrm{H}, 5-\mathrm{CH}_{2}\right), 5.41(\mathrm{~s}, 2 \mathrm{H}$, $\left.17-\mathrm{CH}_{2}\right), 6.52(\mathrm{~s}, 1 \mathrm{H}, 20-\mathrm{OH}), 7.26(\mathrm{~s}, 1 \mathrm{H}, 14-\mathrm{CH}$,$) ,$ $7.47 \sim 7.51(\mathrm{~m}, 2 \mathrm{H}, 9-\mathrm{CH}, 11-\mathrm{CH}), 8.06$ (d, $J=9.00 \mathrm{~Hz}$, 1H, 12-CH), 8.52 (s, 1H, 7-CH); ESI-MS m/z: $494.1(\mathrm{M}+$ $\mathrm{H}^{+}$); HRMS calcd for $\mathrm{C}_{26} \mathrm{H}_{28} \mathrm{~N}_{3} \mathrm{O}_{7}[\mathrm{M}+\mathrm{H}]^{+}$494.1922, found 494.1930 .

3.2.4 9-硝基-10-差基喜树碱氨基酸缀合物 $\mathbf{5 a} \sim \mathbf{5 b}$ 的合成

$100 \mathrm{mg}$ (0.19 mmol)化合物 3 于圆底烧瓶中, 溶于 3 $\mathrm{mL}$ DMSO, 加入 1.5 倍量 $L$-氨基酸类试剂, 外浴 $80{ }^{\circ} \mathrm{C}$ 下反应 $8 \mathrm{~h}$. 反应完全后, 自然冷却至室温, 将反应液慢 慢滴加到 $40 \mathrm{~mL}$ 冰水中, 过滤得到黄色固体粗品. 粗品 用甲醇/氯仿混合溶剂溶清, 柱层析 [Silica Gel H, 洗脱 剂: $V$ (二氯甲烷) $: V($ 甲醇 $)=30: 1]$, 分得目标化合物 $5 \mathbf{a}$ 和 $5 \mathbf{b}^{[13]}$.

9-硝基-10-羊基喜树碱脯氨酸缀合物(5a): 淡黄色 固体, m.p. 229 232 ${ }^{\circ} \mathrm{C}$, 收率 6.50\%. ${ }^{1} \mathrm{H}$ NMR (DMSO$\left.d_{6}, 400 \mathrm{MHz}\right) \delta: 0.86\left(\mathrm{t}, J=7.24 \mathrm{~Hz}, 3 \mathrm{H}, 18-\mathrm{CH}_{3}\right), 1.80 \sim$ $2.20\left(\mathrm{~m}, 6 \mathrm{H}, 19-\mathrm{CH}_{2}, 4^{\prime}-\mathrm{CH}_{2}\right.$ and $\left.3{ }^{\prime}-\mathrm{CH}_{2}\right), 3.11 \sim 3.23(\mathrm{~m}$, $2 \mathrm{H}, 5 '-\mathrm{CH}_{2}$ and $\left.\mathrm{OCH}_{2} \mathrm{CH}_{2}\right), 4.30(\mathrm{t}, J=7.82 \mathrm{~Hz}, 1 \mathrm{H}$, $\left.2^{\prime}-\mathrm{CH}\right), 4.48 \sim 4.66\left(\mathrm{~m}, 2 \mathrm{H}, 5^{\prime}-\mathrm{CH}_{2}{ }^{\prime}\right.$ and $\left.\mathrm{OCH}_{2} \mathrm{CH}_{2}{ }^{\prime}\right), 4.68$ (brs, $\left.2 \mathrm{H}, \mathrm{OCH}_{2} \mathrm{CH}_{2}\right), 5.25\left(\mathrm{~s}, 2 \mathrm{H}, 5-\mathrm{CH}_{2}\right), 5.41(\mathrm{~s}, 2 \mathrm{H}$, 17- $\mathrm{CH}_{2}$ ), 6.54 (s, 1H, 20-OH), 7.32 (s, 1H, 14-CH), 8.07 $(\mathrm{d}, J=9.78 \mathrm{~Hz}, 1 \mathrm{H}, 11-\mathrm{CH}), 8.44$ (d, $J=9.39 \mathrm{~Hz}, 1 \mathrm{H}$, 12-CH), 8.44 (s, 1H, 7-CH), 8.95 (brs, 1H, COOH); ESI-MS $m / z$ : $551.8\left(\mathrm{M}+\mathrm{H}^{+}\right)$; HRMS calcd for $\mathrm{C}_{27} \mathrm{H}_{27} \mathrm{~N}_{4} \mathrm{O}_{9}[\mathrm{M}+\mathrm{H}]^{+}$551.1773, found 551.1775.

9-硝基-10-羊基喜树碱酪氨酸甲酯缀合物 $(\mathbf{5 b})$ : 淡 黄色固体, m.p. $137 \sim 143{ }^{\circ} \mathrm{C}$, 收率 $26.30 \%$. ${ }^{1} \mathrm{H}$ NMR (DMSO- $\left.d_{6}, 400 \mathrm{MHz}\right) \delta: 0.86$ (t, $J=7.04 \mathrm{~Hz}, 3 \mathrm{H}$, $\left.18-\mathrm{CH}_{3}\right), 1.83 \sim 1.87\left(\mathrm{~m}, 2 \mathrm{H}, 19-\mathrm{CH}_{2}\right), 2.21$ (brs, $\left.1 \mathrm{H}, \mathrm{NH}\right)$, 2.72 (brs, $2 \mathrm{H}, \mathrm{NHCH}_{2}$ ), $2.77 \sim 2.91\left(\mathrm{~m}, 2 \mathrm{H}, \mathrm{PhCH}_{2}\right), 3.51$ (s, 4H, NHCHCOOCH 3 ), 4.37 (brs, 2H, 10-- $\mathrm{OCH}_{2}$ ), 5.25 (s, 2H, 5- $\left.\mathrm{CH}_{2}\right), 5.41\left(\mathrm{~s}, 2 \mathrm{H}, 17-\mathrm{CH}_{2}\right), 6.54(\mathrm{~s}, 1 \mathrm{H}, 20-\mathrm{OH})$, 6.59 (d, $\left.J=7.82 \mathrm{~Hz}, \mathrm{Ph}-3^{\prime}, 5^{\prime}-\mathrm{CH}\right), 6.91$ (d, $J=8.21 \mathrm{~Hz}$, Ph-2',6'-CH), 7.31 (s, 1H, 14-CH), 7.99 (d, J=9.39 Hz, $1 \mathrm{H}, 11-\mathrm{CH}), 8.39$ (d, $J=9.39 \mathrm{~Hz}, 1 \mathrm{H}, 12-\mathrm{CH}), 8.43$ (s, 1H, 7-CH), 9.15 (s, 1H, PhOH); ESI-MS $m / z: 631.3\left(\mathrm{M}+\mathrm{H}^{+}\right)$; HRMS calcd for $\mathrm{C}_{32} \mathrm{H}_{30} \mathrm{~N}_{4} \mathrm{NaO}_{10}[\mathrm{M}+\mathrm{Na}]^{+}$653.1854, found 653.1841 .

\section{3 生物活性测试}

以 10-羊基喜树碱作为阳性对照物, 采用 CCK-8 法, 分别测试了 10 -差基喜树碱氨基酸缀合物 $\mathbf{4 a} \sim \mathbf{4 g}$ 和 9硝基-10-羊基喜树碱氨基酸缀合物 $\mathbf{5 a} \sim \mathbf{5 b}$ 对人口腔鳞 癌细胞 $\mathrm{KB}$ 、人肝癌细胞 $\mathrm{HepG} 2$ 和小鼠结肠癌细胞 $\mathrm{C} 26$ 三组细胞株的生长抑制情况.

取对数生长期的肿瘤细胞，用 $0.25 \%$ 胰酶消化，离 心后加培养基调整细胞浓度为 4 万个 $/ \mathrm{mL}$, 然后接种入 96 孔培养板, 每孔 $100 \mu \mathrm{L}$. 待测化合物用 DMSO 稀释, 取 $1 \mathrm{uL}$ 加入 96 孔板后得以下梯度浓度: 50000, 5000, $500,50,5$ 和 $0.5 \mathrm{nmol} / \mathrm{L}$ 等 6 个受试浓度, 每个浓度均设 3 个复孔, 阴性对照为等体积 $(1 \mu \mathrm{L})$ 药物溶剂(DMSO), 同时设清零孔对照. 细胞于接种 $12 \mathrm{~h}$ 待其贴壁后加药, 每孔 $1 \mu \mathrm{L}$ (阴性对照组和清零孔加 $1 \mu \mathrm{L}$ DMSO). 加药后 细胞置 $37{ }^{\circ} \mathrm{C}, 5 \% \mathrm{CO}_{2}$ 、饱和湿度培养 $48 \mathrm{~h}$, 然后每孔加 入 $10 \mu \mathrm{L} \mathrm{CCK}-8$ 试剂后继续培养 $4 \mathrm{~h}$. 酶标仪检测各孔 在 $450 \mathrm{~nm}$ 处吸光度 $\mathrm{A}_{450}$. 按公式: 细胞增殖抑制率 $(\%)=\left(\right.$ 对照组 $\mathrm{A}_{450}$ 一给药组 $\mathrm{A}_{450} /$ 对照组 $\left.\mathrm{A}_{450}\right) \times 100 \%$, 应用 SPSS 软件计算半数抑制浓度 $\left(\mathrm{IC}_{50}\right.$ 见表 1$)$.

\section{References}

[1] Wall, M. E.; Wani, M. C.; Cook, C. E. J. Am. Chem. Soc. 1966, 88, 3888.

[2] Bart, L. S.; Kathryn, H.; Michael, D. F.; Craig, A. B.; Alex, B. B.; Lance, S. Proc. Natl. Acad. Sci. U. S. A. 2002, 99, 15387.

[3] Raffaella, C.; Loana, M.; Sabrina, D.; Roberto, A.; Stella, T.; Donato, C.; Franco, Z.; Michelandrea, D. C.; Giovanni, L. B.; Nadia, Z. Eur. J. Med. Chem. 2013, 63, 387

[4] Inhee, C.; Choonmi, K.; Sun, C. Arch. Pharm. Res. 2007, 30, 1526.

[5] John, E. K.; Daniel, S. P. Biochemistry 2001, 40, 9792.

[6] Fan, Y.; Weinstein, J. N.; Kohn, K. W.; Shi, L. M.; Pommier, Y. J. Med. Chem. 1998, 41, 2216.

[7] Chu, W. China New Med. 2003, 2, 76 (in Chinese). (禇伟，中国新医药, 2003, 2, 76.)

[8] William, G.; Vishal, P.; Joshua, C. C.; Li, P. Y.; Rebecca, H.; Adegboyega, K. O. Bioorg. Med. Chem. Lett. 2013, 23, 3283.

[9] Yang, C. H.; Schneider, E.; Kuo, M. L.; Volk, E. L. Biochem. Pharmacol. 2000, 60, 831.

[10] Ye, D. Y.; Shi, Q.; Leung, C. H.; Kim, S. W.; Park, S. Y.; Elizabeth, A. G.; Jiang, Z. L.; Zhu, H.; Morris-Natschke, S. L.; Cheng, Y. C.; Lee, K. H. Bioorg. Med. Chem. 2012, 20, 4489.

[11] Song, Y. L.; Zhang, W. N.; Ji, H. T. Chin. Phim. J. 2002, 37, 646 (in Chinese).

(宋云龙, 张万年, 季海涛, 中国药学杂志, 2002, 37, 646.) 
[12] Venditto, V. J.; Simanek, E. Mol. Pharm. 2010, 7, 307.

[13] Joan, B.; Tomas, R.; Josep, D. Bioorg. Med. Chem. Lett. 1999, 9, 1859.

[14] Stella, V. J.; Himmelstein, K. J. J. Med. Chem. 1980, 23, 1275.

[15] Naka, T.; Kubo, Y.; Nishikawa, K. Drug Des. Discovery 1999, 16, 95.

[16] http://www.molinspiration.com/cgi-bin/properties
[17] Penta, A.; Ganguly, S.; Murugesan, S. Inter. J. Pharm. Pharm. Sci. 2013, 5, 659 .

[18] Meng, G. R.; Li, J. J.; Zhang, Q.; Ma, H. M. Chin. J. Org. Chem. 2012, 32, 2378 (in Chinese).

(孟光荣, 李嘉俊, 张倩, 马红梅, 有机化学, 2012, 32, 2378.)

[19] Wani, M. C.; Nicholas, A. W.; Wall, M. E. J. Med. Chem. 1986, 29, 2358.

(Zhao, X.) 